\title{
Valor agregado en la educación de la Universidad Nacional de Colombia
}

\author{
Added Value in Education by \\ the National University of Colombia
}

\section{Juan Campos Torres, Cristian Carrillo Cárdenas, Miguel Otero Ceballos y Mario Risueño Rueda*}

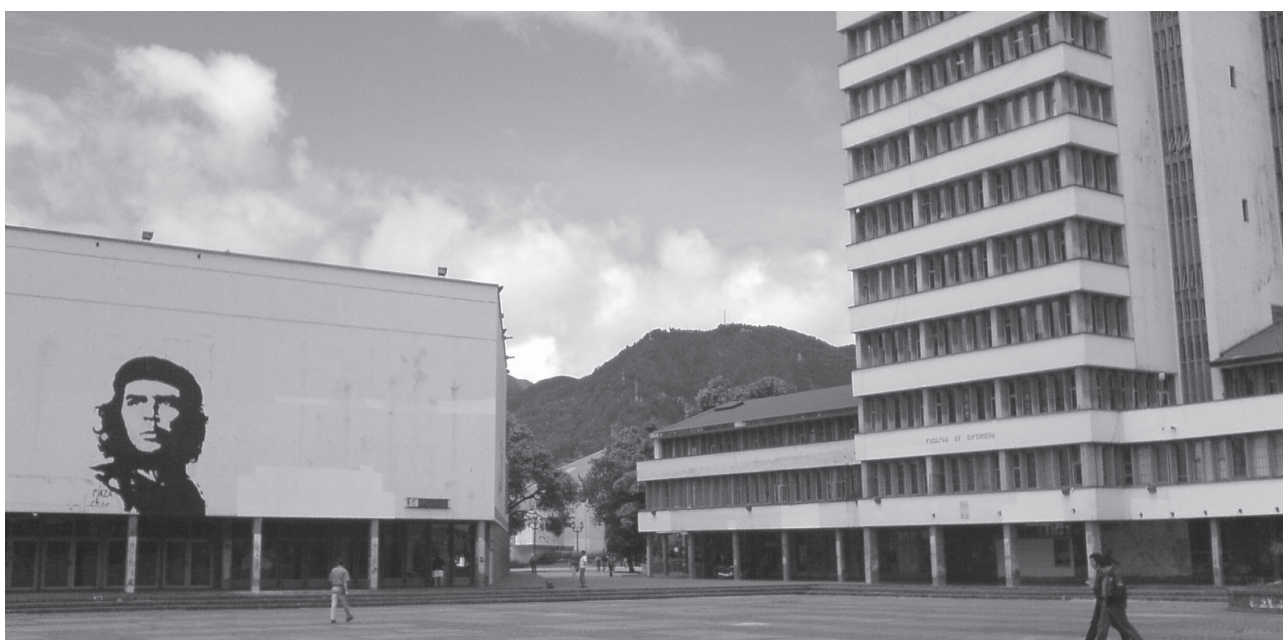

\section{Resumen}

El propósito de este trabajo investigativo es determinar el valor agregado de los programas impartidos en las escuelas de la Facultad de Ciencias económicas de la Universidad Nacional (sede Bogotá), tomando como referencia el componente de lectura crítica de las pruebas Saber Pro llevadas a cabo entre los años 2008-2015. Para ello, se propone un enfoque metodológico de carácter mixto (cuantitativo-cualitativo), tomando variables de tipo demográfico, socioeconómico e institucionales, las cuales permitirán develar qué tan significativo es el aporte académico que brinda tal universidad a la sociedad (en el campo educativo), así como la calidad de conocimientos que poseen sus agresados en términos del componente citado.

Palabras clave: valor agregado, lectura crítica, educación, Universidad Nacional, pruebas Saber Pro.

Citar este artículo como: Campos Torres, J., Carrillo Cárdenas, C., Otero Ceballos, M. y Risueño Rueda, M.. (2017). Valor agregado en la educación de la Universidad Nacional de Colombia. Revista Papeles, 9(17), 107-118.

Fecha de recibido: marzo 30 de 2017.

Fecha de aceptación: junio 5 de 2017.

* Estudiantes de la Facultad de Economía de la Universidad Nacional. Este trabajo fue producto de la investigación al interior del seminario de Metodología de la Investigación, bajo la dirección del profesor Enrique Ferrer-Corredor. Correos: jacampost@unal.edu.co, cccarrilloc@unal.edu.co, maoteroc@unal.edu.co, mfrisuenor@unal.edu.co 


\begin{abstract}
The main purpose of this research is to determine the added value of the majors offered by the Faculty of Economical Sciences at the National University (at the Bogotá campus), taking as a main reference the component of critical reading in the Saber Pro exams developed between 2008 and 2015. Thus it is proposed a mix methodological approach (cualitative-cuantitative), using demographic, socioeconomic and institutional variables, which will allow us to reveal how meaningful is the academical contribution offered by that university to the society (in the educational field), and just like the quality of the knowledge acquired by its graduates (in terms of the component proposed).
\end{abstract}

Keywords: added value, critical reading, education, National University, Saber Pro exams.

\title{
Introducción
}

La educación superior en América Latina experimentó, en la década de 1990, un marcado interés por la calidad educativa, ya que se comenzó a reconocer en ella la principal herramienta para responder a las exigencias de un mundo globalizado, con crecientes demandas de mayor y mejor preparación de las personas (Vargas y Guiselle, 2007, p. 4). Como efecto colateral de esta globalización, de las políticas liberales y de la escasa regulación fomentadas por el consenso de Washington, se creó una gran cantidad de instituciones de educación superior ${ }^{1}$, particularmente de carácter privado, acarreando un detrimento de la calidad de la educación impartida en este nivel formativo (Fernández, 2004).

Como efecto colateral de esta
globalización, de las políticas liberales
y de la escasa regulación fomentadas
por el consenso de Washington,
se creó una gran cantidad de
instituciones de educación superior,
particularmente de carácter privado,
acarreando un detrimento de la
calidad de la educación impartida en
este nivel formativo.

Pero ya mucho antes de este proceso de pérdida de calidad, se buscaban factores asociados al rendimiento académico (Rodríguez y Galván, 2016, p. 72), a fin de ofrecer herramientas que permitieran evaluar el desempeño estudiantil y la calidad de la educación impartida (Vargas y Guiselle, 2007, p. 6). El problema es que el concepto de calidad educativa resulta muy relativo, ya que sus múltiples definiciones reflejan perspectivas y necesidades específicas, con lo cual termina siendo terriblemente subjetivo ${ }^{2}$.

En esta investigación se ha optado por el criterio de valor agregado, el cual evalúa las competencias adquiridas entre dos momentos específicos, atendiendo tanto a variables académicas como extraescolares (Gonzales y Espinoza, 2008, p. 10; Rodríguez y Galván, 2016, p. 68).

1 Para el caso América Latina, en la segunda mitad del siglo $\mathrm{XX}$, el número de instituciones y la matrícula anual experimentaron un incremento sostenido y en la década de 1990 creció un 6\% ( $8 \%$ sector privado y 2,5 sector público [Vargas y Guiselle, 2007, p. 5]).

2 La calidad es de naturaleza similar a la belleza, términos ambos muy relativos, ya que cada uno depende del criterio del evaluador, lo que termina generando subjetividad en el juzgamiento (Gonzales y Espinoza, 2008, p. 5). 


\section{Marco teórico}

El valor agregado da cuenta de la contribución de una institución al progreso de sus estudiantes, habiendo eliminado la influencia de factores ajenos que pueden contribuir a dicho progreso (Rodríguez y Galván, 2016, p. 74). Para realizar tal cálculo se hacen inferencias estadísticas sobre la eficacia de las instituciones educativas, a través del seguimiento de la trayectoria de los estudiantes, analizando los resultados de dos o más años (Rodríguez y Galván, 2016, p. 73). Esto permite evaluar la eficacia de manera mucho más acertada y justa que con el simple resultado promedio de los estudiantes (Rodríguez y Galván, 2016, p. 73), lo cual, más aún, no permitiría hacer comparaciones validas entre instituciones ${ }^{3}$.

El mejor criterio para determinar la eficacia educacional es el valor que la educación de una institución agrega a los atributos iniciales de los estudiantes (Creemers, 1997, citado en Rodríguez y Galván, 2016, p. 71). Este modelo de valor agregado correlaciona la eficacia académica institucional con el progreso educativo del estudiante, pero partiendo del supuesto de que los estudiantes cuentan con unos antecedentes en términos de logro académico, habilidad innata, aptitud para el aprendizaje, de grupo de estudio y un contexto familiar y social que necesariamente influyen en sus resultados (Rodríguez y Galván, 2016, p. 71).

En este sentido, en Colombia, la educación se mide con pruebas estandarizadas para los diferentes niveles educativos, diseñadas y desarrolladas por el Ministerio de Educación y por el ICFES. Estas evaluaciones permiten conocer el desempeño académico y la calidad de las instituciones educativas. Sin embargo, aún no se ha generalizado la práctica de evaluar el aporte de las universidades, en los que se consideren estudios longitudinales (lo que se aprende durante el tránsito por la universidad); lo que permitiría separar las contribuciones de estas instituciones y los factores contextuales ajenos al control de las aulas (Rodríguez y Galván, 2016, p. 71).

\section{Objetivos de lainvestigación}

En esta investigación se busca determinar el valor agregado por los programas impartidos en las escuelas de la facultad de ciencias económicas de la sede Bogotá de la Universidad
Nacional, a saber: Administración de Empresas, Contaduría Pública y Economía, focalizados en el componente de lectura crítica, entre los años 2008-2015.

\section{Objetivo general}

Determinar el diferencial en el componente de lectoescritura, comparando los resultados obtenidos en los exámenes de admisión de la Universidad Nacional y en las pruebas Saber Pro, logrados por los estudiantes de la

3 Se pueden establecer como criterios para decir que una institución es mejor que otra cuando se comparan si son homólogas en sus fines, concordantes en su misión y se encuentran en un contexto similar.
Facultad de Ciencias Económicas de la sede Bogotá que ingresaron en el período comprendido entre 2008 y 2010 y que presentaron sus exámenes Saber Pro aproximadamente entre los años 2012 y 2015; con el fin de evaluar los aportes que hizo la Universidad Nacional de Colombia en la formación de sus educandos en el aspecto subrayado y de esta manera determinar el valor agregado por tal institución en ese aspecto. 


\section{Objetivos específicos}

Recopilar los puntajes de los estudiantes de la Facultad en los exámenes de admisión a la Universidad Nacional en el período comprendido entre 2008 y 2010 a través de los informes consolidados de la Universidad Nacional:

- Obtener los puntajes obtenidos por los estudiantes de la Facultad de Ciencias Económicas en las pruebas Saber Pro en el período comprendido entre 2012 y 2015 a través de los informes consolidados de la Universidad Nacional.

- Realizar los tratamientos estadísticos necesarios para poder hacer comparaciones viables entre estos dos tipos de pruebas y de esta manera obtener algún tipo de conclusión de los resultados.
- Comparar la información de los puntajes obtenidos por los estudiantes de la Facultad en los exámenes de admisión a la Universidad Nacional y en las pruebas Saber Pro en el período comprendido entre 2008 y 2015.

- Recopilar la información que permita determinar los aspectos socioeconómicos y socioculturales de los estudiantes que conforman la facultad y analizar cuáles de estos pudieron haber influido en los resultados.

- Analizar las tendencias obtenidas en las pruebas, relacionando factores como el programa curricular de procedencia, el género de los estudiantes y cualquier otro aspecto que se considere relevante para explicar los resultados.

\section{Marco conceptual}

\section{Metodología}

El logro académico se define como los aprendizajes reales que han adquirido los estudiantes durante un periodo académico, al que se identificará como valor agregado del aprendizaje. Este valor se obtiene de restarle el logro previo (Exámenes de admisión) al logro bruto (Saber Pro), a partir de los porcentajes de estudiantes que se ubicaron en los mejores quintiles de las respectivas pruebas tenidas en consideración (adaptado de Rodríguez y Galván 2016, p. 85):

$$
\mathrm{VA}=\mathrm{LB}-\mathrm{LP}
$$

$$
\begin{aligned}
\mathrm{VA} & =\text { Valor agregado) } \\
\mathrm{LB} & =\text { Saber Pro) } \\
\mathrm{LP} & =\text { Exámenes Admisión) }
\end{aligned}
$$

Pero adicional a esto, la construcción del modelo requiere analizar la influencia del contexto universitario y familiar, el cual termina impactando el rendimiento académico. Es decir que además de las variables cuantitativas se deberían incluir variables de orden institucional y personal. Verbigracia: requisitos de ingreso, requisitos entre materias, nivel de formación de los profesores (Rodríguez y Galván, 2016, p. 74), nivel educativo de los padres, nivel socioeconómico, entre muchos otros que se escapan al alcance de esta investigación (Bosa, 1970).

Por este motivo, la investigación buscó -aunque de manera imperfecta- un enfoque mixto: de un lado, el enfoque cuantitativo daría cuenta de los resultados obtenidos en las pruebas de admisión y las pruebas Saber Pro. De otro lado, en el enfoque cualitativo, analizaría la influencia de otras variables, como el nivel socioeconómico y sociocultural o la institucionalidad universitaria; cuestión que no se logró a plenitud, por lo reducido del alcance de la presente investigación.

A partir de esto, la meta es establecer un modelo de tipo correlacional que debería permitir determinar el efecto de las variables consideradas sobre los resultados obtenidos en las pruebas Saber Pro. Con este propósito, se recolectó la información necesaria tanto en la sede de la Facultad, como en las publicaciones 
que la Universidad Nacional de Colombia realiza con el fin de mantener informados a los interesados de los resultados de su gestión educativa. El proceso investigativo se llevó a cabo en tres etapas, atendiendo y condensando los objetivos específicos:

En la primera se determinó el logro académico de los estudiantes utilizando como insumo los resultados en los exámenes de admisión y las pruebas Saber Pro, tomando como referente el componente de lectura crítica.

\section{Limitaciones de la investigación}

Una limitante inicial de la investigación fue el acceso a la información institucional de la Facultad, proceso que presentó muchos inconvenientes y obstáculos, y, más significativo aún, fue el excesivo tiempo de espera para recibir la información solicitada. Otra de las limitantes significativas fue la imposibilidad de crear un modelo que incluyera
En la segunda etapa se calculó el valor agregado teniendo en cuenta el algoritmo propuesto y partiendo de los porcentajes de los estudiantes ubicados en cada quintil establecido en las pruebas.

En la tercera y última etapa se buscó determinar el impacto sobre el desempeño estudiantil, de las diferentes variables que se tuvieron en cuenta a lo largo del proceso investigativo y se contrastaron las hipótesis planteadas al inicio del proyecto con los resultados obtenidos. estadísticamente las variables cualitativas de la investigación, denominadas "otras variables a considerar", las cuales fueron simplemente mencionadas como posibles factores influyentes de los resultados, pero sin pruebas definitivas de tal correlación. Esto se debió a que los datos suministrados por las escuelas fueron muy sintéticos y para nada discriminaron la información de los estudiantes.

\section{Población y muestra}

La población corresponde a los estudiantes que presentaron los exámenes de admisión, esperando ingresar a los programas de Administración de empresas, Economía y Contaduría pública, en la Facultad de Ciencias económicas de la Universidad Nacional, sede Bogotá, entre los años 2008 y 2010, y los que presentaron las pruebas Saber Pro entre los años 2012 y 2015. A continuación, se detalla el número de aspirantes a ingresar a la facultad y el número que presentó las pruebas Saber Pro y algunas características institucionales de la UN (nota: todos los gráficos son tomadas de la Oficina Nacional de Planeación Universidad Nacional, 2013):

Tabla 1. Número de aspirantes a la facultad.

\begin{tabular}{|c|c|c|c|c|c|c|}
\hline Año & $\mathbf{2 0 0 8 - 1}$ & $\mathbf{2 0 0 8 - 2}$ & $\mathbf{2 0 0 9 - 1}$ & $\mathbf{2 0 0 9 - 2}$ & $\mathbf{2 0 1 0 - 1}$ & $\mathbf{2 0 1 0 - 2}$ \\
\hline Número aspirantes & 2.937 & 2.751 & 2.969 & 2.822 & 3.340 & 3.275 \\
\hline
\end{tabular}

Fuente: Oficina Nacional de Planeación Universidad Nacional (2013).

Tabla 2. Número de estudiantes en pruebas Saber Pro.

\begin{tabular}{|l|c|c|c|c|c|c|c|c|}
\hline \multicolumn{1}{|c|}{ Año } & $\mathbf{2 0 1 2 - 1}$ & $\mathbf{2 0 1 2 - 3}$ & $\mathbf{2 0 1 3 - 1}$ & $\mathbf{2 0 1 3 - 3}$ & $\mathbf{2 0 1 4 - 1}$ & $\mathbf{2 0 1 4 - 3}$ & $\mathbf{2 0 1 5}-\mathbf{1}$ & $\mathbf{2 0 1 5 - 3}$ \\
\hline Administración & 50 & 38 & 43 & 76 & 7 & 98 & 125 & 9 \\
\hline Contaduría & 61 & 52 & 41 & 79 & 8 & 97 & 104 & 1 \\
\hline Economía & 62 & 26 & 43 & 69 & 11 & 81 & 106 & 4 \\
\hline
\end{tabular}

Fuente: Oficina Nacional de Planeación Universidad Nacional (2013). 


\section{Hipótesis de la investigación}

Con respecto a la investigación, se plantearon algunas hipótesis previas sobre los resultados que esta podría arrojar:

1. La variable género no será tan influyente en la investigación como sí lo pueden ser la edad de estudiante, su nivel socioeconómico y cultural, entre otros, es decir, el género no afectará los resultados.

2. La carrera seleccionada será un factor determinante en los resultados obtenidos en las pruebas Saber Pro; esto teniendo en cuenta el nivel de exigencia en el componente de lectoescritura que demanda cada carrera.

3. Otras variables que tendrán mayor impacto en los resultados obtenidos en las pruebas Saber Pro serán las relacionados con el nivel socioeconómico del grupo familiar, así como el nivel cultural del mismo, ya que esto facilitaría los procesos de aprendizaje.

\section{Otras variables a considerar en la investigación}

\section{Aspectos demográficos}

Población estudiantil por sexo y por edad

Gráfica 1. Distribución de los estudiantes por género.

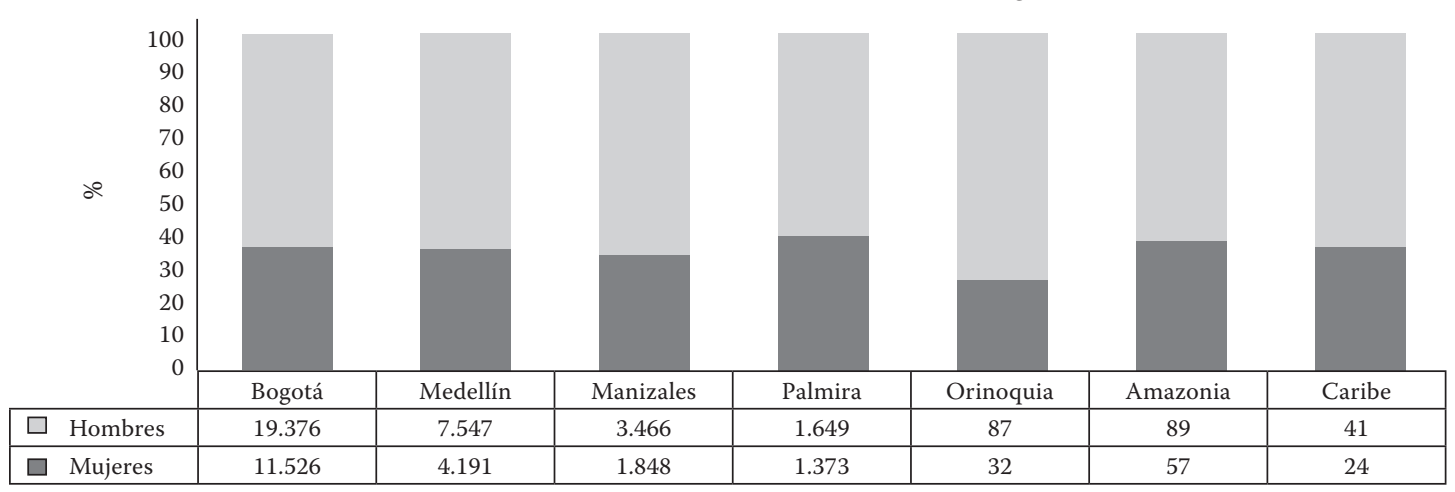

Fuente: elaboración propia.

Gráfica 2. Clasificación estudiantes por género y por edad.

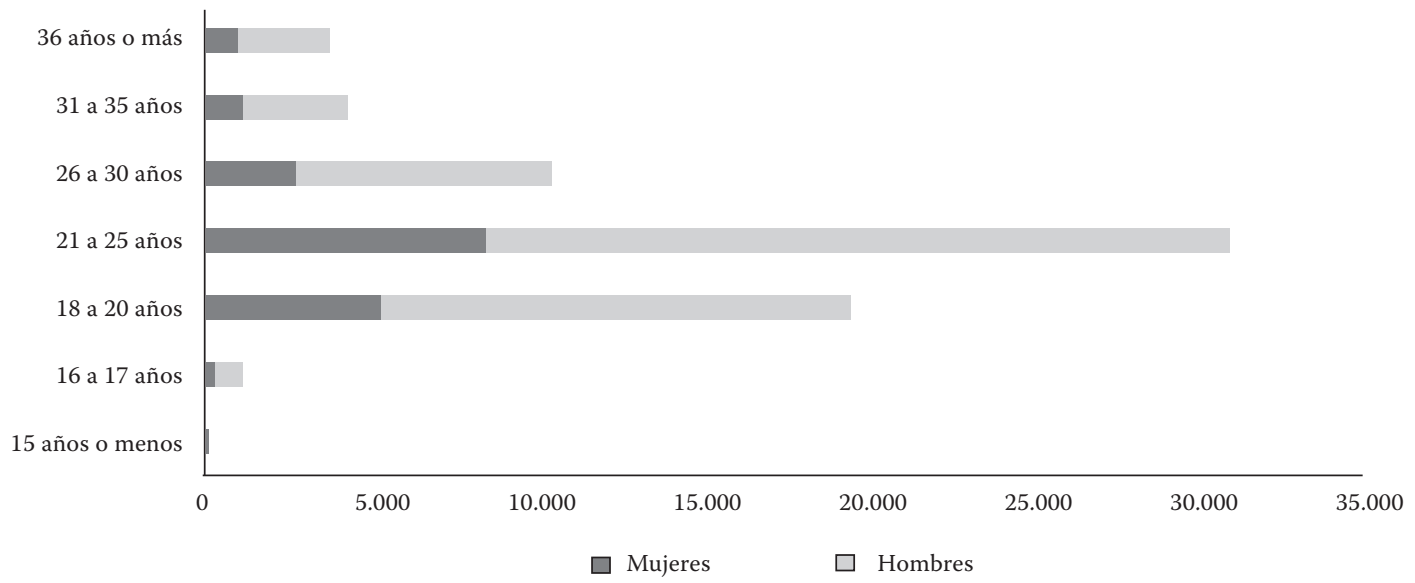

Fuente: elaboración propia. 
Como resulta evidente, en la población estudiantil de la Universidad Nacional de Colombia se observa una mayoría leve, pero sostenida, de la población masculina en todas las sedes, y más marcada aún en la Orinoquía.
Por otro lado, la edad predominante está entre los 18 y 25 años, con cierta significancia del grupo de los 25 a los 30 años, es decir, una población mayormente joven y con una leve mayoría masculina.

\section{Aspectos socioeconómicos}

\section{Clasificación socioeconómica de los estudiantes}

Gráfica 3. Estratificación económica de los estudiantes de la UN.

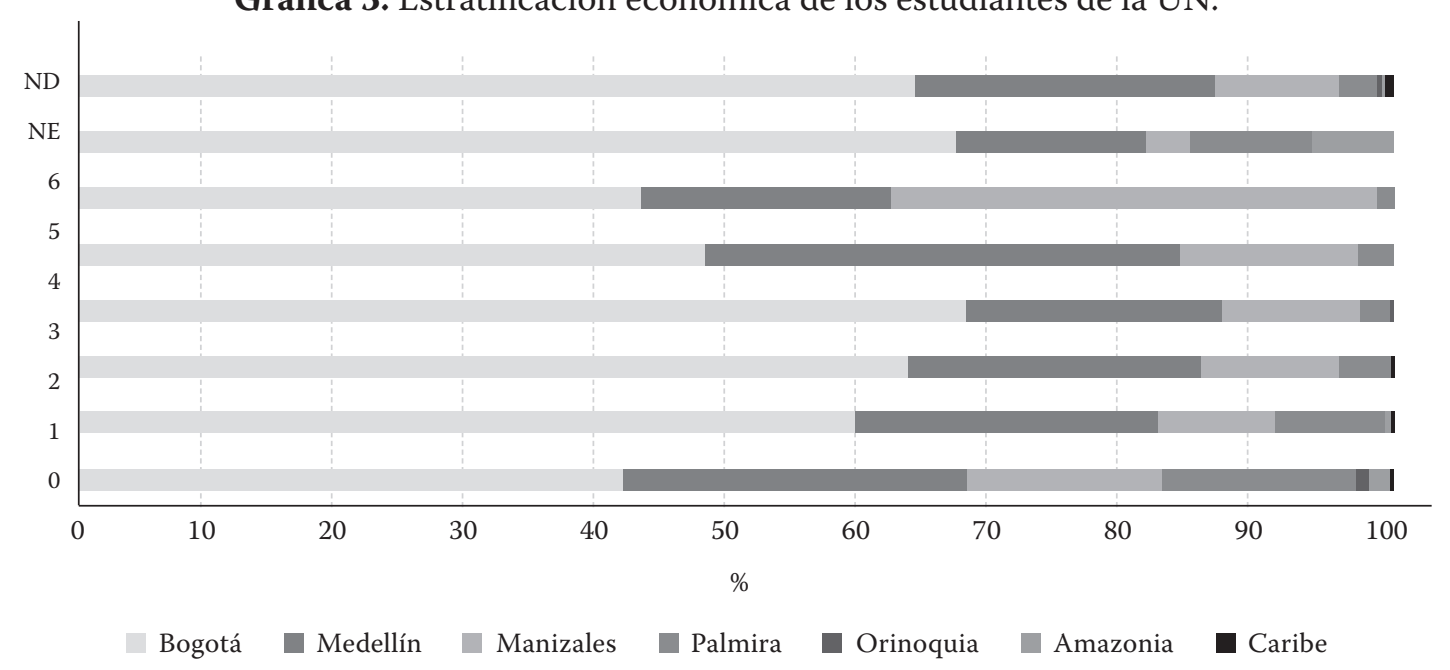

Fuente: elaboración propia.

Tabla 3. Estratificación económica estudiantes UN (sede Bogotá).

\begin{tabular}{|c|c|c|c|c|c|c|c|c|}
\hline \multirow{2}{*}{ Sede } & \multicolumn{8}{|c|}{ Estrato socioeconómico 2013 } \\
\cline { 2 - 10 } & $\mathbf{1}$ & $\mathbf{2}$ & $\mathbf{3}$ & $\mathbf{4}$ & $\mathbf{5}$ & $\mathbf{6}$ & $\mathbf{N E}$ & ND \\
\hline Bogotá & 1.718 & 9.784 & 12.173 & 3.437 & 527 & 130 & 126 & 3.007 \\
\hline
\end{tabular}

Fuente: elaboración propia.

Gráfica 4. Graduados por estrato socioeconómico.

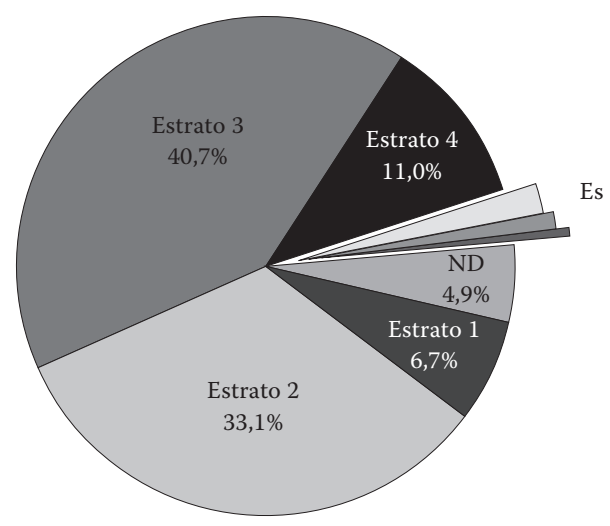

Fuente: elaboración propia.
Tabla 4. Graduados por estrato socioeconómico.

\begin{tabular}{|c|r|r|}
\hline $\begin{array}{c}\text { Estrato } \\
\text { Socioeconómico }\end{array}$ & Bogotá & $\mathbf{\%}$ \\
\hline 1 & 146 & 5,0 \\
\hline 2 & 918 & 31,6 \\
\hline 3 & 1.236 & 42,6 \\
\hline 4 & 356 & 12,3 \\
\hline 5 & 65 & 2,2 \\
\hline 6 & 15 & 0,5 \\
\hline NE & 13 & 0,4 \\
\hline ND & 152 & 5,2 \\
\hline Total & 2.901 & 100,0 \\
\hline
\end{tabular}

Fuente: elaboración propia. 


\section{Aspectos institucionales}

Nivel de preparación de la planta educativa de UN

Tabla 5. Nivel de preparación de la planta educativa de la UN.

\begin{tabular}{|l|c|c|}
\hline \multicolumn{1}{|c|}{ Docentes por nivel de formación } & Universidad & País \\
\hline Total docentes & 2.890 & 108.093 \\
\hline Docentes con formación en doctorado & 1.170 & 6.398 \\
\hline Participación del total de docentes (\%) & 40 & 6 \\
\hline Participación de los docentes con doctorado de la universidad en el país )\% & \multicolumn{2}{|c|}{18} \\
\hline Docentes con formación en maestría (\%) & 1.331 & 25.738 \\
\hline Participación del total de docentes (\%) & 46 & 24 \\
\hline Participación de los docentes con maestría de la Universidad en el país (\%) & \multicolumn{2}{|c|}{5} \\
\hline
\end{tabular}

Fuente: Dirección Nacional de personal. Universidad Nacional de Colombia (planta de docentes con corte a noviembre de 2012. Los docentes con maestría oncluye 316 con titulo en especialidades médicas)

* Ministerio de Educación Superior, Subdirección de Desarrollo sectorial. Estadisticas (Actualización a 31 de diciembre de 2013) (descarga: 13 de marzo de 2014)

Elaboración propia.

\section{Composición socioetnográfica del estudiantado}

Tabla 6. Composición socioetnográfica de los estudiantes.

\begin{tabular}{|l|r|r|c|c|c|r|}
\hline \multicolumn{7}{|c|}{ Matriculados por primera vez por tipo de admisión } \\
\hline Regular & $\begin{array}{c}\text { Comunidad } \\
\text { Indigena }\end{array}$ & $\begin{array}{c}\text { Mejores bachilleres } \\
\text { de municipios } \\
\text { pobres }\end{array}$ & $\begin{array}{c}\text { Mejores } \\
\text { bachilleres } \\
\text { del país }\end{array}$ & $\begin{array}{c}\text { Mejores bachilleres de } \\
\text { población negra, afrocolombiana, } \\
\text { palenquera y raizal }\end{array}$ & Total \\
\hline Bogotá & 4.826 & 105 & 54 & 49 & 45 & 5.07 \\
\hline Medellín & 2.203 & 47 & 35 & 23 & 14 & 2.332 \\
\hline Manizales & 1.030 & 25 & 14 & 1 & 16 & 1.084 \\
\hline Palmira & 485 & 14 & 18 & 0 & 0 & 533 \\
\hline Orinoquia & 129 & 0 & 0 & 0 & 0 & 129 \\
\hline Amazonia & 140 & 0 & 0 & 0 & 0 & 140 \\
\hline Caribe & 49 & 0 & 0 & 0 & 99 & 49 \\
\hline Total & 8.862 & 191 & 121 & 73 & 1 & 9.346 \\
\hline $\begin{array}{l}\text { (\%) } \\
\text { Matriculados/ } \\
\text { admitidos (\%) }\end{array}$ & 85 & 2 & 1 & 1 & 66 & 100 \\
\hline
\end{tabular}

Fuente: Dirección Nacional de Admisiones. Sistema de Información Académico (SIA). Cálculo oficina nacional de Planeación

\section{Procedencia académica de los estudiantes}

Tabla 7. Procedencia de los aspirantes y admitidos.

\begin{tabular}{|c|c|c|c|c|}
\hline \multicolumn{5}{|c|}{ Aspirantes } \\
\hline Carácter de la institución & 2013-1 & 2013-2 & Total & $\%$ \\
\hline Oficial & 4.628 & 3.081 & 7.709 & 64 \\
\hline Privado & 2.454 & 1.573 & 4.027 & 34 \\
\hline Sin información & 123 & 91 & 214 & 2 \\
\hline Total & 7.205 & 4.745 & 11.950 & 100 \\
\hline \multicolumn{5}{|c|}{ Admitidos } \\
\hline Carácter de la institución & 2013-1 & 2013-2 & Total & $\%$ \\
\hline Oficial & 2.547 & 1.670 & 4.217 & 82 \\
\hline Privado & 190 & 677 & 867 & 17 \\
\hline Sin información & & 34 & 34 & 1 \\
\hline Total & 2.737 & 2.381 & 5.118 & 100 \\
\hline
\end{tabular}

Fuente: elaboración propia. 


\section{Desarrollo de lainvestigación}

De acuerdo con lo planteado en la metodología de la presente investigación, el proceso se llevó a cabo en tres etapas, las cuales respondieron a los objetivos específicos esbozados con anterioridad. Estas etapas se describen a continuación:

\section{Primera etapa. Recopilación de la información}

Respecto de los resultados en el componente de lectura crítica obtenidos, primero por los aspirantes a ingresar a la facultad en los exámenes de admisión entre el 2008 y el 2010 y luego por los estudiantes de la UN que presentaron las pruebas Saber Pro entre los años 2012 y 2015, se determinó el logro académico de los estudiantes y se clasificó a los estudiantes por su nivel de desempeño, establecido en quintiles:

Tabla 8. Proporción estudiantes en cada quintil por año / Exámenes de admisión.

\begin{tabular}{|l|r|r|r|r|r|r|r|r|r|r|r|r|}
\cline { 2 - 13 } \multicolumn{1}{c|}{} & \multicolumn{4}{c}{ Admisiones 2008 } & \multicolumn{3}{c|}{ Admisiones 2009 } & \multicolumn{3}{c|}{ Admisiones 2010 } \\
\hline Quintil 1 & 74 & $2,52 \%$ & 3 & $0,11 \%$ & 37 & $1,25 \%$ & 29 & $1,03 \%$ & 111 & $3,32 \%$ & 37 & 1,13 \\
\hline Quintil 2 & 353 & $12,02 \%$ & 246 & $8,94 \%$ & 1.313 & $44,22 \%$ & 445 & $15,77 \%$ & 1.105 & 33,08 & 1,191 & $36,37 \%$ \\
\hline Quintil 3 & 888 & $30,23 \%$ & 1.688 & $61,36 \%$ & 1.214 & $40,89 \%$ & 1.173 & $41,77 \%$ & 1.900 & 56,89 & 1.821 & $36,37 \%$ \\
\hline Quintil 4 & 1.202 & $40,93 \%$ & 705 & 25,63 & 397 & $13,37 \%$ & 1.075 & $38,09 \%$ & 218 & $6,53 \%$ & 220 & $6,72 \%$ \\
\hline Quintil 5 & 420 & $14,30 \%$ & 109 & $3,96 \%$ & 8 & $0,27 \%$ & 100 & $3,54 \%$ & 6 & $0,18 \%$ & 6 & $0,18 \%$ \\
\hline & 2.937 & $100 \%$ & 2.751 & $100 \%$ & 2.969 & $100 \%$ & 2.822 & $100 \%$ & 3.340 & $100 \%$ & 3.275 & $100 \%$ \\
\hline
\end{tabular}

Fuente: elaboración propia.

Tabla 9. Proporción de los estudiantes en cada quintil por año/Pruebas Saber Pro.

\begin{tabular}{|c|c|c|c|c|c|c|c|c|c|c|c|c|c|c|c|c|c|c|c|c|c|c|c|c|}
\hline \multirow[b]{3}{*}{ Quintil 1 } & \multicolumn{6}{|c|}{ 2012-1 } & \multicolumn{6}{|c|}{ 2012-3 } & \multicolumn{6}{|c|}{ 2013-2 } & \multicolumn{6}{|c|}{ 2013-3 } \\
\hline & \multicolumn{2}{|c|}{ Admon } & \multicolumn{2}{|c|}{ Contad } & \multicolumn{2}{|c|}{ Economía } & \multicolumn{2}{|c|}{ Admon } & \multicolumn{2}{|c|}{ Contad } & \multicolumn{2}{|c|}{ Economía } & \multicolumn{2}{|c|}{ Admon } & \multicolumn{2}{|c|}{ Contad } & \multicolumn{2}{|c|}{ Economía } & \multicolumn{2}{|c|}{ Admon } & \multicolumn{2}{|c|}{ Contad } & \multicolumn{2}{|c|}{ Economía } \\
\hline & 0 & $0 \%$ & 2 & $3,28 \%$ & 1 & $1,61 \%$ & 1 & $2,36 \%$ & 0 & $0 \%$ & 1 & $3,85 \%$ & 0 & $0 \%$ & 1 & $2,44 \%$ & 0 & 0 & 0 & $0 \%$ & 2 & $2,53 \%$ & 0 & $0 \%$ \\
\hline Quintil 2 & 2 & $4 \%$ & 1 & $1,64 \%$ & 5 & $8,06 \%$ & 0 & $0 \%$ & 1 & $1,92 \%$ & 2 & $7,69 \%$ & 0 & $0 \%$ & 0 & $0 \%$ & 2 & $4,65 \%$ & 1 & $1,32 \%$ & 2 & $2,53 \%$ & 3 & $4,35 \%$ \\
\hline Quintil 3 & 2 & $4 \%$ & 3 & $4,92 \%$ & 7 & $11,29 \%$ & 2 & 5,26 & 2 & $3,85 \%$ & 3 & $11,54 \%$ & 2 & $6,98 \%$ & 2 & $4,88 \%$ & 5 & $11,63 \%$ & 6 & $7,89 \%$ & 7 & $8,86 \%$ & 9 & $13,04 \%$ \\
\hline Quintil 4 & 7 & $14 \%$ & 14 & $22,95 \%$ & 11 & $17,74 \%$ & 10 & $26,32 \%$ & 17 & $32,69 \%$ & 4 & $15,38 \%$ & 5 & $16,28 \%$ & 5 & $12,2 \%$ & 14 & $32,56 \%$ & 6 & $7,89 \%$ & 7 & $8,86 \%$ & 24 & $34,78 \%$ \\
\hline \multirow[t]{2}{*}{ Quintil 5 } & 39 & $78 \%$ & 41 & $67,21 \%$ & 38 & $61,29 \%$ & 25 & $65,79 \%$ & 32 & $61,54 \%$ & 16 & $61,54 \%$ & 33 & 76,74\% & 33 & $80,49 \%$ & 22 & $51,16 \%$ & 63 & $82,89 \%$ & 61 & $77,22 \%$ & 33 & $47,83 \%$ \\
\hline & 50 & $100 \%$ & 61 & $100 \%$ & 62 & $100 \%$ & 38 & $100 \%$ & 52 & $100 \%$ & 26 & $100 \%$ & 41 & $100 \%$ & 41 & $100 \%$ & 43 & $100 \%$ & 76 & $100 \%$ & 79 & $100 \%$ & 69 & $100 \%$ \\
\hline
\end{tabular}

\begin{tabular}{|c|c|c|c|c|c|c|c|c|c|c|c|c|c|c|c|c|c|c|c|c|c|c|c|c|}
\hline \multirow[b]{3}{*}{ Quintil 1 } & \multicolumn{6}{|c|}{ 2014-2 } & \multicolumn{6}{|c|}{ 2014-3 } & \multicolumn{6}{|c|}{ 2015-2 } & \multicolumn{6}{|c|}{ 2015-3 } \\
\hline & \multicolumn{2}{|c|}{ Admon } & \multicolumn{2}{|c|}{ Contad } & \multicolumn{2}{|c|}{ Economía } & \multicolumn{2}{|c|}{ Admon } & \multicolumn{2}{|c|}{ Contad } & \multicolumn{2}{|c|}{ Economía } & \multicolumn{2}{|c|}{ Admon } & \multicolumn{2}{|c|}{ Contad } & \multicolumn{2}{|c|}{ Economía } & \multicolumn{2}{|c|}{ Admon } & \multicolumn{2}{|c|}{ Contad } & \multicolumn{2}{|c|}{ Economía } \\
\hline & 0 & $0 \%$ & 0 & $0 \%$ & 0 & $0 \%$ & 0 & $0 \%$ & 0 & $0 \%$ & 1 & $1,23 \%$ & 0 & $0 \%$ & 2 & 0,01923 & 1 & $0,94 \%$ & 2 & $22,22 \%$ & 0 & $0 \%$ & 0 & $0 \%$ \\
\hline Quintil 2 & 0 & $0 \%$ & 0 & $0 \%$ & 1 & $9,09 \%$ & 1 & $1,02 \%$ & 1 & $1,03 \%$ & 10 & $12,35 \%$ & 1 & $0,8 \%$ & 3 & 0,02885 & 4 & $3,77 \%$ & 2 & $22,22 \%$ & 0 & $0 \%$ & 1 & $25 \%$ \\
\hline Quintil 3 & 0 & $0 \%$ & 0 & $0 \%$ & 1 & $9,09 \%$ & 4 & $4,08 \%$ & 6 & $6,19 \%$ & 19 & $23,46 \%$ & 12 & $9,6 \%$ & 7 & 0,06731 & 20 & $18,87 \%$ & 1 & $11,11 \%$ & 0 & $0 \%$ & 2 & $50 \%$ \\
\hline Quintil 4 & 1 & $14,29 \%$ & 0 & $0 \%$ & 4 & $36,36 \%$ & 8 & $8,16 \%$ & 17 & $17,53 \%$ & 19 & $23,46 \%$ & 13 & $10,40 \%$ & 14 & 0,13462 & 30 & $28,30 \%$ & 3 & $33,33 \%$ & 1 & $100 \%$ & 1 & $25 \%$ \\
\hline Quintil 5 & 6 & $85,71 \%$ & 8 & $100 \%$ & 5 & $45,45 \%$ & 85 & $86,73 \%$ & 73 & $75,26 \%$ & 32 & $39,51 \%$ & 99 & \begin{tabular}{|l|}
$79,20 \%$ \\
\end{tabular} & 78 & 0,75 & 51 & $48,11 \%$ & 1 & $11,11 \%$ & 0 & $0 \%$ & 0 & $0 \%$ \\
\hline & 7 & $100 \%$ & 8 & $100 \%$ & 11 & $100 \%$ & 98 & $100 \%$ & 97 & $100 \%$ & 81 & $100 \%$ & 125 & $100 \%$ & 104 & $100 \%$ & 106 & $100 \%$ & & $100 \%$ & 1 & $100 \%$ & 4 & $100 \%$ \\
\hline
\end{tabular}

Fuente: elaboración propia. 


\section{Segunda etapa. Evaluación de los resultados}

Gracias a la clasificación en quintiles, se puede observar que en las pruebas de admisión los estudiantes ubicados en el quintil 5 no superan el 3\% (a excepción del 2008, cuando fue el 14\%, y que sigue siendo muy bajo). El mayor número de estudiantes se ubicó en los quintiles 2 y 3, y un porcentaje algo considerable en el quintil 4 , pero que no logra ser significativo ni sostenido en todos los años evaluados. Por otro lado, en las pruebas Saber Pro, el porcentaje de estudiantes ubicados en el máximo quintil es superior, de manera sistemática y sostenida, en casi todos los años al 75\% y en algunos periodos alcanza casi el 90\%. De igual forma, a lo largo del periodo evaluado, más del $90 \%$ de los estudiantes se ubica en los quintiles 4

Tabla 10. Cálculo de valor agregado.

\begin{tabular}{|c|c|c|c|}
\hline Año & $\begin{array}{c}\text { \% saber } \\
\text { pro }\end{array}$ & $\begin{array}{c}\text { \% } \\
\text { Asmisión }\end{array}$ & $\begin{array}{c}\text { Diferencial } \\
\text { (Valor agregado) }\end{array}$ \\
\hline $2008 / 12$ & $78.00 \%$ & $14.30 \%$ & $63.70 \%$ \\
\hline $2009 / 13$ & $76.74 \%$ & $0.27 \%$ & $76.47 \%$ \\
\hline $2010 / 14$ & $85.71 \%$ & $0.18 \%$ & $85.53 \%$ \\
\hline $2008 / 15$ & $79.20 \%$ & $0.18 \%$ & $79.02 \%$ \\
\hline
\end{tabular}

Fuente: elaboración propia. y 5, es decir, los niveles superiores de desempeño, lo cual refleja el excelente nivel logrado por los estudiantes al finalizar sus estudios universitarios.

Esto ya es un indicativo muy diciente del aporte que la educación de la Universidad Nacional hace a sus estudiantes, ya que la gran mayoría entró con un desempeño medio-bajo, de acuerdo a sus resultados en las pruebas de admisión, pero al hacer su tránsito por la universidad, sus resultados mejoraron ostensiblemente en las pruebas Saber Pro, llegando a un nivel superior en un porcentaje muy alto.

Ahora, si, finalmente, haciendo uso del algoritmo y atendiendo solo a la proporción de los estudiantes ubicados en el quintil 5 y bajo el supuesto de que los estudiantes hicieron su carrera universitaria en el tiempo previsto de cinco años, se puede obtener un cálculo del valor agregado restando del porcentaje de estudiantes ubicados en el quintil 5 de las pruebas Saber Pro, el porcentaje de los ubicados en el mismo quintil, de las pruebas de admisión cinco años atrás:

\section{Tercera etapa. Contrastación de los resultado}

No fue posible una integración efectiva de las variables de tipo cualitativo que se intentaron tener en consideración para el modelo de valor agregado planteado, ya que no se dispuso de información suficientemente discriminada para generar conclusiones acertadas sobre tales aspectos y establecer correlaciones explicativas de los resultados. Los datos proporcionados por las sedes de las escuelas de la facultad fueron muy sintéticos y superficiales; no había clasificación por género y ni siquiera por opción de carrera en el caso del examen de admisión.

En cuanto a las variables de nivel socioeconómico, nivel sociocultural y características institucionales, no fue tampoco posible obtener información específica sobre la población que atañe a esta investigación. La información recolectada fue de carácter general para toda la universidad y fue citada en el trabajo a manera de contextualización de la situación general de los estudiantes. Esto impidió establecer correlaciones significativas entre estas variables y los resultados obtenidos de valor agregado por la universidad.

Sin embargo, los aportes de otras investigaciones y documentos recopilados sí permiten hacer inferencias respecto de la correlación de estas variables con los resultados de logro académico o valor agregado. Estas conclusiones se mencionarán en la sección final del documento. 
En cuanto a las hipótesis planteadas al inicio de la investigación, y como resultado de lo planteado en los párrafos anteriores, no fue posible contrastar de manera definitiva la veracidad de las mismas. El género de los aspirantes y los estudiantes que presentaron las pruebas Saber Pro no fue suministrado por la sede; lo mismo sucede con los aspectos socioeconómicos y culturales.
Respecto de la hipótesis de que el pénsum de la carrera afectaría los resultados obtenidos en las pruebas Saber Pro, esta no parece tener asidero, ya que la contrastación de los resultados muestra desempeños muy similares y parejos entre las dos escuelas de las que fue posible obtener información.

\section{Resultados y discusiones}

- Con base a los porcentajes obtenidos de los estudiantes clasificados en los quintiles 4 y 5 , de acuerdo al desempeño en los exámenes de admisión y las pruebas Saber Pro, es posible afirmar que los primeros muestran mayor heterogeneidad y menor consistencia que los segundos, en donde se puede observar un mayor aglutinamiento de los resultados en los quintiles superiores. Además, también se puede observar que el desempeño del grupo fue mejor en la prueba Saber Pro que en las pruebas de admisión.

- En una investigación de la Universidad de Antioquia, en la que se buscó analizar cómo los procesos institucionales, la dirección de los colegios y las circunstancias del colegio y de sus estudiantes pueden afectar el rendimiento en la prueba de estado para el ingreso a la educación superior, se concluyó que cuando el capital humano es superior, el rendimiento se eleva al igual que cuando la institución enfatiza y revisa los procesos metodológicos, pedagógicos y los conceptos de enseñanza que desarrolla el docente (Tobón, Posaday Ríos, 2009, citado en Marco Tulio Rodríguez Sandoval, 2016, p.72).

- En algunos trabajos sobre la escuela secundaria que analizaban los efectos de la composición socioeconómica y de ciertos procesos institucionales, se concluyó que no todo es atribuible a los factores individuales del alumno o su clasificación socioeconómica, y que la magnitud de tal efecto es bastante más reducida que las expectativas de algunos discursos sobre efectividad escolar (Cervini, 2003, p. 92, citado en Sandoval, 2016, p.72).

- A pesar de los avances que se han dado en los últimos años, la medición por valor agregado sigue quedándose escasa al estimar el efecto de los factores ajenos a la institución, ya que al solo incluir datos de resultados exante y expost se estaría estimando incorrectamente el efecto de las instituciones educativas (Rodríguez y Galván, 2016, p. 73).

\section{Aportes personales y conclusiones}

Contrario a lo que se había pensado al inició de la investigación, el aporte de valor agregado de la UN es muy significativo. Recuérdese que la hipótesis planteaba que por la calidad de los estudiantes que ingresan a dicha institución, los cambios no serían tan notorios al finalizar sus carreras, tal como sucede, por ejemplo, con la Universidad de los Andes, donde sus alumnos no muestran mayores niveles de valor agregado luego de finalizado su paso por la universidad.

De igual manera sucede con los estudiantes de la Universidad de Antioquia y de la Universidad Pedagógica Nacional, que actualmente se ubican en los primeros lugares de valor agregado en el componente de lectura crítica, 
es decir, universidades públicas con altísimos niveles de exigencia de entrada y durante la carrera, pero no necesariamente con los estudiantes con los mejores entornos socioeconómicos y culturales.

Estos ejemplos permiten inferir, por tanto, que el impacto de las variables socioeconómicas y culturales no influyen tan significativamente como podría esperarse, ya que estas deberían impactar positiva o negativamente en la disposición de las personas a dedicar más tiempo y esfuerzo y ser foco en mayor o menor medida de distracciones. Sin embargo, las variables institucionales sí parecen tener un rol más significativo en el logro académico de los estudiantes, tal como sucede con las universidades públicas, las cuales tienes altos estándares para la admisión de sus nuevos alumnos y, de igual manera, las exigencias a lo largo de la carrera también son notables.

No obstante lo anterior, el nivel de empleabilidad de las universidades privadas de gran prestigio sigue siendo muy superior al de las universidades públicas, ya que los empleadores parecen moverse más por el prestigio de la institución de procedencia, que por los niveles de logro de sus alumnos.

\section{Referencias}

Bosa, G. (1970). Rendimiento académico y satisfacción estudiantil: Una evaluación del sistema universitario mexicano. Revista Mexicana De Sociología, 32, 1005-1042.

Gonzales, L. E. y Espinoza, O. (2008). Calidad de la educación superior. Calidad de la educación No 28, 32.

Oficina Nacional de Planeación Universidad Nacional. (2013). Estadísiticas e indicadores de la Universidad Nacional. Estadísticas e indicadores de la Universidad Nacional No 19, 147.
Rico Higuita, D. A. (2005). Perfil socioeconómico de los estudiantes. Oficina de Planeación Universidad Nacional de Colombia, 79.

Rodríguez, R. R. (2015). Medición del valor agregado para la educación superior en Bogotá. Bogotá: Universidad Santo Tomas.

Rodríguez, S. M. y Galván, M. A. (2016). Estudio del valor agregado. Escenarios, 19, 67-82.

Vargas, G., y Guiselle, M. (2007). Factores asociados al rendimiento académico en estudiantes universitarios. Educación, 31(22). 